Check for updates

Cite this: RSC Adv., 2017, 7, 29575

Received 3rd May 2017

Accepted 12th May 2017

DOI: 10.1039/c7ra04951j

rsc.li/rsc-advances

\section{Amphotericin B-loaded mannose modified poly(D,L- lactide-co-glycolide) polymeric nanoparticles for the treatment of visceral leishmaniasis: in vitro and in vivo approaches}

\begin{abstract}
Santanu Ghosh, (D) Suman Das, Asit Kumar De, Nabanita Kar and Tanmoy Bera*
It is evident from many previous research works that the mannose receptors on the surface of macrophages play an important role in the process of phagocytosis and they are over-expressed in some intracellular parasitic diseases like leishmaniasis. Toxicity and drug resistance were the major drawbacks in the treatment of visceral leishmaniasis in the past. To overcome these setbacks we have developed amphotericin B-loaded mannose modified PLGA nanoparticles. O-Stearoyl mannose was synthesized and used to prepare mannose modified PLGA nanoparticles. FTIR and DSC analyses were carried out to determine the drug-excipient compatibility and particle size, and SEM and TEM studies were carried out to determine the physicochemical characteristics of the formulated nanoparticles. An in vitro cumulative drug release study for the formulated nanoparticles was carried out, showing the release of amphotericin B in a controlled manner for a prolonged period of time. From the ex vivo cellular uptake study it was observed that the rate of intracellular internalization was significantly higher for the mannose-modified nanoparticles than for the unmodified ones. Selectivity indexes were calculated to determine the antileishmanial efficiency in the in vitro cellular amastigote model, and were almost 4 fold higher for the mannose modified formulation than for the free amphotericin B. A mice model was used for an in vivo study, showing a $98.19 \% \pm 0.33 \%$ suppression of the amastigote burden in the spleen. So, it could be concluded that amphotericin B-loaded mannose modified PLGA nanoparticles are more efficacious in the treatment of visceral leishmaniasis in both in vitro and in vivo models than unmodified nanoformulations
\end{abstract}

\section{Introduction}

Leishmaniasis, a disease associated with poverty, ${ }^{1}$ is known to be a vector borne disease. The female phlebotomine sandfly is the vector, which transmits the promastigote phase of the obligate parasite to the biological systems of humans, resulting in one of the three existing forms of leishmaniasis: cutaneous, mucocutaneous or visceral. Among them visceral leishmaniasis $(\mathrm{VL})$ is the most fatal condition of the pathogenesis, with some characteristic symptoms known to be irregular fever, weight loss, hepatomegaly, splenomegaly and anaemia. The endemicity of the disease, especially VL, in countries like India, Bangladesh, Ethiopia, Sudan, Brazil, etc. proves that poverty is strongly associated with the advancement of the disease. However, nowadays it is not restricted to those endemic countries but has extended its claws to non-endemic European

Laboratory of Nanomedicine, Division of Pharmaceutical Biotechnology, Department of Pharmaceutical Technology, Jadavpur University, 188 Raja S.C. Mallick Road, Kolkata-700 032, West Bengal, India. E-mail: proftanmoybera@gmail.com; Tel: +91 9831470041 countries as well. International travelling, migration from the affected countries and HIV co-infections have triggered this. ${ }^{2-4}$

For the development of new drug substances or therapeutic strategies against leishmaniasis, it is important to know the life cycle of the obligate parasite in humans. Following the bite of an infected female sandfly, flagellated promastigotes are transmitted into the biological fluid of humans. Macrophages are recruited at the site of infection and phagosomal vacuoles are formed during the process of phagocytosis. This is followed by the association of the lysosomes situated inside the macrophages, and phagolysosomal vacuoles are developed. Inside the phagolysosomal vacuoles it is a very hostile environment for any pathogens to merely survive, however, leishmania is among a few that can survive as well as multiply in this antagonistic environment. Therefore, macrophages act as a safe house for the promastigotes where they transform into amastigotes and multiply. When the amastigote burden increases inside the macrophages, it bursts and releases the amastigotes to infect other macrophages. Although macrophages are known to be the most important component of the process of phagocytosis, neutrophils also play an important role. Infection initiated by 
the bite of a sand fly influences the neutrophils to be recruited to the site of infection. ${ }^{5,6}$ According to Laufs et al. ${ }^{7}$ and van Zandbergen et al. ${ }^{8}$ as the life period of the neutrophils is very short, they just perform as 'Trojan horses', which means the parasites use the neutrophils for avoiding the cell activation phenomenon to enter into the macrophages. During the interaction with the parasite, receptors such as mannose receptors, CR1 and CR3 complement receptors and fibronectin receptors on the surface of macrophages play an important role. ${ }^{9}$ Among the other receptors, many scientists have revealed the importance of mannose receptors for macrophage-targeted drug delivery. ${ }^{10-12}$

Although in the last few decades many remarkable research works regarding the development of a newer delivery system have been carried out, intra-macrophage drug delivery is still very challenging. Parameters like the size and shape of the polymeric nanoparticles always play a key role in the processes of biodistribution, cellular uptake and target specificity of drugs used for their intended purpose. Nanodisks, nanorods, plateloids, ellipsoids, nanoneedles, and filamentous or worm-like micelles are different shapes of nanoformulations currently in the limelight. ${ }^{13}$ In a recent review article, Truong et al. demonstrated the advantages of filomicelles over the conventional spherical polymeric nanoparticle in the field of anticancer drug delivery. ${ }^{13,14}$ According to Bera et al., in the case of macrophageassociated drug delivery, spherical particles in the size range of around $200 \mathrm{~nm}$ could effectively be phagocytosed by the macrophages. ${ }^{15}$ However, as present drug delivery systems are not selective for intracellular drug delivery, the chemotherapeutics result in unwanted toxicities at their dosing regimen. Therefore, a significant effort is necessary to develop a delivery system which can increase the efficiency of the chemotherapeutics as well as decrease their undesirable toxicities.

VL infections result in over-expressed mannose receptors (MMRs; CD206) and other receptors such as scavenger receptors and stearylamine receptors, ${ }^{\mathbf{1 6}, 17}$ which can bind to pathogenassociated molecular patterns (PAMPs). Among the mannose receptors, MMR is a transmembrane glycoprotein composed of a type-II fibronectin domain, an N-terminal cysteine-rich domain and eight lectin-like C-type carbohydrate recognition domains. ${ }^{18}$ A wide variety of infectious pathogens contain mannose, fucose and $N$-acetylglucosamine as PAMPs on their cell surface, which can bind with the extracellular carbohydrate recognition domains of the macrophages. ${ }^{19}$ Thus, overexpressed mannose receptors, especially MMRs, could be targeted by developing a mannose associated drug delivery system where mannose acts as the PAMP. In addition, this mannose associated drug delivery to the macrophages could generally occur by receptor-mediated endocytosis, using the mannose receptor in the case of VL. ${ }^{20}$

Many research works have been carried out where polyethylene glycol was used as a spacer between the ligand, mannose and the drug encapsulated polymer for this kind of drug delivery system, but it is expected that the flexible long chains of polyethylene glycol could shield the mannose, making them unable to interact with the mannose receptor. ${ }^{\mathbf{2 1}}$ Therefore we attached a stearoyl moiety to the mannose to synthesize $O$ stearoyl mannose, which might circumvent this problem.

At the present time, the treatment of endemic VL is restricted to a few chemotherapeutic agents like paromomycin, miltefosine, amphotericin B, etc. Toxicity is a major concern for all of them, however drug resistance makes it more complicated. Nephrotoxicity and ototoxicity are associated with paromomycin therapy, and teratogenicity is the major problem for orally active miltefosine. ${ }^{22}$ Slow intravenous administration of amphotericin B is needed to overcome its toxicity. However, amphotericin B is still the gold standard for the treatment of VL.

So, in this experimental work we have prepared amphotericin B-loaded $O$-stearoyl mannose-associated poly(D,L-lactide-coglycolide) nanoparticles, which may have a significantly increased rate of uptake by the macrophages, could provide controlled release of amphotericin $\mathrm{B}$ for an extended period of time and could efficiently treat VL by decreasing the parasite burden significantly inside the spleen.

\section{Materials and methods}

\subsection{Materials}

Poly(D,L-lactide-co-glycolide) [PLGA acid terminated RG $502 \mathrm{H}$, $M_{\mathrm{w}}$ 7000-17 000], amphotericin B (AmB), paromomycin (PMM), medium 199, fetal bovine serum and RPMI 1640 were purchased from Sigma-Aldrich, India. Fluorescein isothiocyanate $98 \%$ (FITC), was procured from Himedia Laboratories. Polyvinyl alcohol (PVA, $M_{\mathrm{w}}$ approx. 125000 ) was purchased from SDFCL, India. Tween 80 was purchased from Merck, India. Sodium stibogluconate (SSG) was a gift from Albert David Ltd. (Kolkata, India). Dichloromethane (DCM) and dimethyl sulfoxide (DMSO) were procured from Merck, India. All other chemicals used were of analytical grade.

\subsection{Ethics statement for animal experiments}

To carry out the animal experiments in this work, BALB/c mice (weighing 20-25 g) of either sex were used. All of the animal experiments were performed in compliance with the relevant laws of the Committee for the Purpose of Control and Supervision of Experiments on Animals (CPCSEA), Govt. of India, India and the University Grants Commission (UGC), New Delhi, India. The Animal Ethics Committee Jadavpur University (AEC/ PHARM/1403/2014) approved the experimental protocols. The procedures were followed in accordance with the committee's guidelines. Polypropylene cages were used to house the animals and they were fed a standard diet and water ad libitum. The mice were exposed to a normal day and night cycle.

\subsection{Synthesis of $O$-stearoyl mannose (OSM)}

$O$-Stearoyl mannose was synthesized by Zhu et $a .^{21}$ with few modifications (Fig. 1). $3.33 \mathrm{mmol}$ D-mannose was dissolved in $15 \mathrm{ml}$ of anhydrous dichloromethane (DCM) in a flat bottomed flask. To the resultant solution, $2.1 \mathrm{ml}$ of anhydrous pyridine and $1.5 \mathrm{ml}$ of anhydrous DCM containing $0.545 \mathrm{mmol}$ stearoyl chloride was subsequently added and this was stirred for $16 \mathrm{~h}$. The reaction mixture was then dispersed into $10 \mathrm{ml}$ of brine 


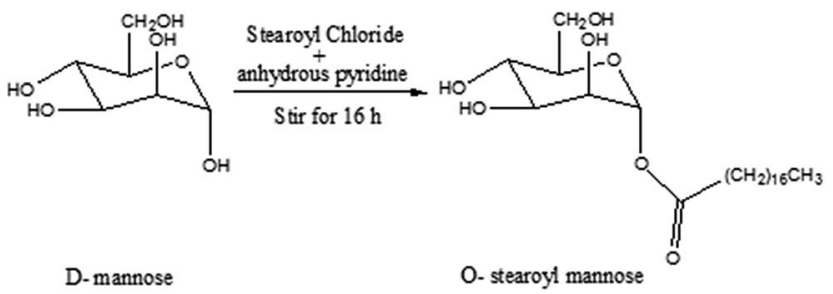

Fig. 1 Synthesis scheme for $O$-stearoyl mannose.

solution in a separating funnel. After separation the DCM layer was collected and anhydrous disodium sulfate was added to it and kept overnight in a closed system. After that the DCM solution was filtered out and evaporated in a water bath, and characterization of the synthesized product was carried out by Fourier transform infrared spectroscopy (FTIR) and ${ }^{1} \mathrm{H}$ nuclear magnetic resonance $\left({ }^{1} \mathrm{H}\right.$ NMR) study.

\subsection{Preparation of nanoparticles}

According to Table 1, three different types (different drug : polymer ratio) of AmB-loaded PLGA nanoparticles were prepared by a double emulsion-solvent evaporation method. ${ }^{23}$ Briefly, weighed amounts of PLGA and AmB (Table 1) were dissolved in $2 \mathrm{ml}$ of DCM. $0.5 \mathrm{ml}$ of $2.5 \%$ aqueous PVA solution was added drop-wise into it during homogenization by a high speed homogenizer (Homogenizer-IKa T10 basic ultra turrax) at an optimized speed of $22000 \mathrm{rpm}$, forming a w/o type emulsion. Then this primary emulsion was added gradually to $75 \mathrm{ml}$ of $1.5 \% \mathrm{w} / \mathrm{v}$ aqueous PVA solution with continuous homogenization (Homogenizer-IKa T10 basic ultra turrax) at $22000 \mathrm{rpm}$ for $5 \mathrm{~min}$ to produce a w/o/w type emulsion. The formed secondary emulsion was stirred on a magnetic stirrer $(2 \mathrm{MLH}$, Remi) overnight to remove the DCM.

Nanoparticles were collected by a cold centrifuge (Sigma $3 \mathrm{~K} 30$, Germany) at high speed. First larger particles were removed by centrifugation at $5000 \mathrm{rpm}$ for $5 \mathrm{~min}$. The supernatant was collected and recentrifuged at $16000 \mathrm{rpm}$ for $40 \mathrm{~min}$. The separated solid nanoparticles were washed thrice with Milli-Q water (Millipore Corp., Billerica, MA, USA) to remove the excess PVA and the unloaded free $\mathrm{AmB}$ and were kept at $-80{ }^{\circ} \mathrm{C}$ in a freezer.

Then the nanoparticles were lyophilized for 7-8 h (Lyophilizer: Instrumentation India, Kolkata, India) followed by storage at $4{ }^{\circ} \mathrm{C}$ for further use.

During the preparation of AmB-loaded PLGA-OSM nanoparticles (ASMN), a weighed amount of OSM (Table 1) was dissolved in DCM along with PLGA and AmB. ${ }^{24}$ Blank nanoparticles were prepared using the same technique except the drugs were not added and in the case of FITC-loaded PLGA-PVA nanoparticles preparation, FITC was used instead of AmB using a similar method of nanoparticle preparation.

\subsection{Characterization of nanoparticles}

2.5.1. Fourier transform spectroscopy (FTIR). Determination of the drug-excipient interaction was done by Fourier transform infrared spectroscopy. PLGA, PVA, AMB, a physical mixture of PLGA + PVA + AMB, a physical mixture of PLGA + PVA + AMB + OSM, blank nanoparticles, AMB loaded PLGA nanoparticles and AMB loaded PLGA-OSM nanoparticles were subjected to obtain the FTIR spectra. Samples were mixed with potassium bromide at a ratio of $1: 100$ before analysis. FTIR was done by FTIR, Model: Nicolet iS10, Thermo Fisher Scientific, USA.

2.5.2. Differential scanning calorimetry (DSC) and thermogravimetric analysis (TGA). DSC and TGA studies were carried out to evaluate the drug-excipient compatibility further (DSC, Model no.: Pyris Diamond TG/DTA, Make: PerkinElmer, Singapore). DSC and TGA of PLGA, AmB, a physical mixture of PLGA + PVA + AmB, a physical mixture of PLGA + PVA + AmB, OSM, blank nanoparticles, AmB-loaded PLGA nanoparticles and AmB-loaded PLGA-OSM nanoparticles were performed in a nitrogen atmosphere $\left(150 \mathrm{ml} \mathrm{min}{ }^{-1}\right)$ using a platinum crucible, with alpha alumina powder used as reference. The temperature range was from $30^{\circ} \mathrm{C}$ to $360{ }^{\circ} \mathrm{C}$.

2.5.3. Particle size, zeta potential and polydispersity index (PDI). The average particle size, zeta potential and PDI were determined by a dynamic light scattering method with data transfer assistance (DTA) software by Zetasizer (Zetasizer Nano ZS90 Model: ZEN3690, Malvern Instruments, Malvern, U.K.) at $25{ }^{\circ} \mathrm{C}$. The samples were prepared by dispersing $2 \mathrm{mg}$ of formulated nanoparticles in $2 \mathrm{ml}$ of Milli-Q water.

2.5.4. Scanning electron microscopy (SEM). The morphology of the nanoparticles was analyzed using a scanning electron microscope (scanning electron microscope JEOL, Tokyo, Japan). Nanoparticles were mounted on stabs and coated with gold (20 nm thickness) using a Sputter Coater JFC1100 , and were observed under the scanning electron microscope.

2.5.5. Transmission electron microscopy (TEM). TEM study was carried out to further evaluate the morphological character (JEM 2100, JEOL, Tokyo, Japan). A diluted suspension of lyophilized nanoparticles was prepared in Milli-Q water. Nanoparticle suspensions were placed onto a carbon-coated

Table 1 The drug polymer ratio, average particle size, zeta potential and polydispersity index of different AmB-loaded PLGA nanoparticles

\begin{tabular}{lllllr}
\hline Formulations & AmB : PLGA : OSM & $\begin{array}{l}\text { \% yield } \\
(\text { mean } \pm \mathrm{SD}, n=3)\end{array}$ & $\begin{array}{l}\text { \% loading } \\
(\text { mean } \pm \mathrm{SD}, n=3)\end{array}$ & $\begin{array}{l}\text { Avg. particle size } \\
(\mathrm{nm})(\mathrm{mean} \pm \mathrm{SD}, n=3)\end{array}$ & $\begin{array}{l}\text { Zeta potential (mV) } \\
(\mathrm{mean} \pm \mathrm{SD}, n=3)\end{array}$ \\
\hline AN1 & $1: 10: 0$ & $70.9 \pm 3.6$ & $6.7 \pm 0.5$ & $182.8 \pm 4.2$ & $-11.8 \pm 2.1$ \\
AN2 & $1: 5: 0$ & $56.4 \pm 2.7$ & $9.0 \pm 0.5$ & $261.2 \pm 5.7$ & $-12.3 \pm 1.8$ \\
AN3 & $3: 10: 0$ & $60.8 \pm 4.1$ & $11.5 \pm 0.7$ & $280.9 \pm 3.6$ & $-14.7 \pm 2.7$ \\
ASMN & $1: 5: 1.5$ & $64.7 \pm 3.4$ & $10.2 \pm 0.8$ & $340.5 \pm 6.2$ & $-24.9 \pm 1.6$
\end{tabular}


copper grid as a thin liquid film by a dropping method. The excess solution was removed by filter paper and dried at room temperature. Soft Imaging Viewer software was used to take TEM images. ${ }^{25}$

2.5.6. AmB loading and entrapment efficiency study. To determine the AmB loading and entrapment efficiency, formulations were dissolved in DMSO at a concentration of $1 \mathrm{mg} \mathrm{ml}^{-1}$ in triplicates. Then a 10 times dilution was made using methanol for each one. The solutions were mixed well and placed in an incubator shaker for $4 \mathrm{~h}$ at $37^{\circ} \mathrm{C}$ followed by centrifugation for $10 \mathrm{~min}$ at $5000 \mathrm{rpm}$. The collected supernatants were used for chromatographic analysis with a C18 phenomenex column $(250 \times 4.6 \mathrm{~mm}, 5 \mu)$. Potassium dihydrogen phosphate buffer of pH 4.0 : acetonitrile $(55: 45)$ was used as the mobile phase and the flow rate was $1.0 \mathrm{ml} \mathrm{min}{ }^{-1}$. The area of AmB was measured at the $\lambda_{\max }$ of $407 \mathrm{~nm}$. The percentage of drug loading and the entrapment efficiency were calculated using the following equations:

Theoretical drug loading $(\%)=$ amount of drug taken to prepare the nanoparticles $\times 100 \%$ /amount of PLGA + amount of drug

Actual drug loading $(\%)=$ amount of drug present in the obtained nanoparticles $\times 100 \%$ /amount of obtained nanoparticles

Drug entrapment efficiency $(\%)=$ actual drug loading $\times 100 \% /$ theoretical drug loading

2.5.7. In vitro drug release study. $2 \mathrm{mg}$ measurements of AmB containing all the different formulations of AmB-loaded nanoparticles were weighed accurately and suspended separately in $2 \mathrm{ml}$ of $0.5 \%$ tween 80 in phosphate buffer saline $(\mathrm{pH}$ 7.4) and kept in a dialysis bag. Then the dialysis bag was placed into a beaker containing $100 \mathrm{ml}$ of the release medium and kept in a shaker bath (BOD-INC-1S, Incon, India) at $37{ }^{\circ} \mathrm{C}$ with constant shaking of $100 \mathrm{rpm}$. After centrifugation, $1 \mathrm{ml}$ of supernatant was collected at scheduled time intervals for fifteen days. The same amount of fresh release medium was replaced. The samples were analyzed at $407 \mathrm{~nm}$ by a similar chromatographic technique as previously mentioned. The drug release mechanism was evaluated by evaluating the release kinetic models such as the zero order, first order, Higuchi, HixsonCrowell and Korsmeyer-Peppas models.

2.5.8. Stability studies. Formulations of $\mathrm{AmB}$ were taken at a concentration of $1 \mathrm{mg} \mathrm{AmB}$ per $\mathrm{ml}$ and kept at $4{ }^{\circ} \mathrm{C}$ and room temperature for 6 months. The sample aliquots were collected at predetermined time points $(1,7,15,30,60,90$ and 180 days) for the determination of particle size and drug loading. ${ }^{16}$

\subsection{Isolation of mice peritoneal macrophages and a trypan blue exclusion assay of macrophage viability}

$\mathrm{BALB} / \mathrm{c}$ mice (20-25 g) were injected with $1.5 \mathrm{ml}$ of sterile thioglycolate medium intraperitoneally to induce non-infectious inflammation. These mice were sacrificed by cervical dislocation after 5 days and peritoneal exudates were harvested by intra-peritoneal lavage. $5 \mathrm{ml}$ of ice-cold RPMI-1640 medium was injected into the peritoneal cavity of the sacrificed mice. The abdominal area was massaged gently for 4-5 min, followed by the withdrawal of the injected fluid by the same syringe. The cell-containing medium was then centrifuged at $4500 \mathrm{rpm}$ for 15 min to collect the cells. Excluding the contaminated erythrocytes, the total number of isolated cells was counted with a hemocytometer. Non-specific esterase staining ${ }^{26}$ confirmed that approximately $60-80 \%$ of the isolated cells were macrophages.

A trypan blue exclusion assay was carried out to determine the isolated macrophages' viability. Briefly, $0.2 \mathrm{ml}$ of isolated cells were aseptically transferred into a $1.5 \mathrm{ml}$ Eppendorf tube and incubated for $3 \mathrm{~min}$ at room temperature with an equal volume of $0.4 \%(\mathrm{w} / \mathrm{v})$ trypan blue solution prepared in $0.81 \%$ sodium chloride and $0.06 \%(\mathrm{w} / \mathrm{v})$ dibasic potassium phosphate. A hemocytometer was used to take the cell count under a light microscope. Viable and non-viable cells were counted separately and the mean counts for three independent cell counts were pooled for analysis. Macrophage cells showed 93\% viability. It was observed that when the macrophage cells were incubated at $45{ }^{\circ} \mathrm{C}$ for $10 \mathrm{~min}$, a viability of only $12 \%$ was noticed.

\subsection{Macrophage uptake study}

Ex vivo cellular uptake of FITC-loaded nanoparticles (FITCloaded PLGA nanoparticles and FITC-loaded PLGA-OSM nanoparticles) was carried out. Macrophages $(200 \mu \mathrm{l})$ were seeded into each culture well containing $800 \mu \mathrm{l}$ of RPMI-1640 medium in such a manner that each of the wells contained 4 $\times 10^{5}$ macrophages. Sterile glass cover slips were placed into each well and macrophages were allowed to adhere onto it in a $5 \% \mathrm{CO}_{2}$ incubator at $37^{\circ} \mathrm{C}$ for $72 \mathrm{~h}$. FITC-loaded nanoparticles of both types were suspended separately in sterile water for injection, and $50 \mu \mathrm{l}$ of those were added to respective wells and allowed to incubate for 10 min under the previous conditions. After that the medium was removed and the cover slips were washed twice with PBS 7.4. The cover slips were removed and placed on glass slides, followed by fixation. They were then observed under a confocal microscope (Olympus Fluoview FV1000, Model: IX81, Singapore) at $60 \times$ magnification and photographs were taken using FV10, version 4.1 software.

\subsection{Parasite culture}

Leishmania donovani AG83 (MHOM/IN/83/AG83), a VL isolate, was gifted by the Indian Institute of Chemical Biology, Council of Scientific and Industrial Research, Kolkata, India. Briefly, $L$. donovani AG83 promastigotes were cultured in $2 \mathrm{mM}$ L-glutamine, $20 \mathrm{mM}$ HEPES, $100 \mu \mathrm{g} \mathrm{ml}^{-1}$ streptomycin, $100 \mathrm{U} \mathrm{ml}^{-1}$ penicillin and $10 \%$ heat inactivated fetal bovine serum supplemented medium 199 at $25{ }^{\circ} \mathrm{C}$. From L. donovani promastigotes the axenic amastigotes were derived by culturing them in MMA/20 (medium for axenic amastigote) at $\mathrm{pH}$ 5.5. Subpassages were done every five days to maintain the culture of the axenic amastigotes and they were kept at $37{ }^{\circ} \mathrm{C}$ in a $5 \% \mathrm{CO}_{2}$ incubator. ${ }^{27}$ 


\subsection{Development of drug resistant amastigote strains}

The technique to develop a drug resistant $L$. donovani strain has already been established. ${ }^{15}$ Briefly, the AG83 wild-type promastigote cells were cultured in medium 199 with supplements in the presence of a drug concentration corresponding to their $\mathrm{IC}_{50}$ value against the particular strain. The drug concentrations were increased after three subcultures. The drugs concentrations were increased in such a way that the total cell population decreased by around $20 \%$ for each of the batches. When $10 \%$ of the initial count of a cell population survived, those particular strains were cultured on medium 199 agar plates in the presence of the same drug concentration. A single colony was selected for further culturing in liquid medium 199. ${ }^{28,29}$ The drug pressure was aloof and the stability was checked at the first, second and fourth months.

\subsection{In vitro growth inhibition study}

An in vitro growth inhibition study against axenic amastigotes of different strains (AG83 wild type, SSG-R, PMM-R and GE1) was carried out by a cell count technique using a hemocytometer. The $50 \%$ inhibitory concentration $\left(\mathrm{IC}_{50}\right)$ of selected drugs was determined by seeding $1 \times 10^{5}$ amastigotes per well in a 96 culture well plate. Drugs were added at their predetermined concentrations. The counts of amastigotes in the drug treated wells were compared with the untreated control. It was presumed that the count of cells in the untreated control represented $100 \%$ growth. The cell counts were taken using the hemocytometer under a light microscope after $72 \mathrm{~h}$ of incubation in a $5 \% \mathrm{CO}_{2}$ incubator at $37^{\circ} \mathrm{C}$. The $\mathrm{IC}_{50}$ values were calculated using the following equation: ${ }^{30}$

$\%$ of inhibition $=($ count of control - count of treated $/$ count of control) $\times 100 \%$

\subsection{Drug susceptibility assay of intracellular amastigotes}

RPMI-1640 medium $(500 \mu \mathrm{l})$ containing macrophage cells $(4 \times$ $10^{5}$ cells per $\mathrm{ml}$ ) was added to each well and incubated in a $5 \%$ $\mathrm{CO}_{2}$ incubator at $37^{\circ} \mathrm{C}$ for $2 \mathrm{~h}$. After $2 \mathrm{~h}, 500 \mu \mathrm{l}$ of warm RPMI1640 medium supplemented with $20 \mathrm{mM}$ L-glutamine, $16 \mathrm{mM}$ $\mathrm{NaHCO}_{3}$, penicillin (50 $\mathrm{U} \mathrm{ml}^{-1}$ ) and streptomycin (50 $\mathrm{mg} \mathrm{ml}^{-1}$ ), and $10 \%$ FCS were added to replace the old media. $0.5 \mathrm{ml}$ of medium $\left(4 \times 10^{6}\right.$ amastigotes per $\left.\mathrm{ml}\right)$ was further added into it. Then the culture plate was kept at $37{ }^{\circ} \mathrm{C}$ in the $5 \% \mathrm{CO}_{2}$ incubator for $4 \mathrm{~h}$ for internalization of the amastigotes into the macrophages. After the period for internalization, fresh RPMI1640 medium, with or without drug or drug encapsulated nanoparticles at the appropriate concentrations, was added in triplicate wells replacing the old media and kept in the $5 \% \mathrm{CO}_{2}$ incubator for $72 \mathrm{~h}$ at $37{ }^{\circ} \mathrm{C}$. After that the medium was removed carefully to recover the cover slips. Then the cover slips were fixed, air dried and stained with Giemsa. Under the microscope, the amastigotes in 100 macrophage cells were counted for each cover slip. Three independent experiments in triplicate were carried out. The results were calculated by calculating the ratio between the infection proportions of untreated and treated macrophage cultures.

\subsection{Assay for toxicity assessment}

2.12.1. In vitro cytotoxicity measurement of isolated macrophages. An MTT assay was employed to measure the cytotoxicity of AmB and AmB-loaded nanoparticles by measuring the mitochondrial activity colorimetrically. The MTT assay was carried out against the isolated macrophages based on the reduction capability of the MTT formazan by the living cells. ${ }^{31}$ The assay was done in 96 adherent culture well plates. $150 \mu \mathrm{l}$ of the supplemented RPMI-1640 medium containing macrophages at a concentration of $10^{4}$ cells per $\mathrm{ml}$ was added to each well. AmB and AmB-loaded nanoparticles were added to the predetermined wells at their predetermined concentrations in triplicates. After $24 \mathrm{~h}$ of exposure in the $5 \% \mathrm{CO}_{2}$ incubator at $37^{\circ} \mathrm{C}, 10 \mu \mathrm{l}$ of MTT at a concentration of $500 \mu \mathrm{g} \mathrm{ml}{ }^{-1}$ was added to each well and kept at the same incubation conditions for $4 \mathrm{~h}$. The MTT was removed and $200 \mu \mathrm{l}$ of DMSO was added to each well to dissolve the purple formazon crystals. The absorbance was measured colorimetrically at $570 \mathrm{~nm}$ by multiwell microplate readers. The results were calculated by calculating the percentage of reduction in the cell viability compared to the untreated control.

2.12.2. In vivo toxicity profiling by biochemical markers. An in vivo toxicity assessment was done to evaluate the hepatic and renal toxicity. Creatinine and blood urea nitrogen were the markers for renal toxicity whereas alanine aminotransferase (ALT) and aspartate aminotransferase (AST) were the markers for the hepatic toxicity assessment. To carry out the study three groups of healthy BALB/c mice (20-25 g) of either sex were taken. Each group contained five mice. Equivalent doses of the nanoformulations of $1 \mathrm{mg}$ of AmB per $\mathrm{kg}$ of body weight were intravenously injected into the tail vein each day for two weeks, whereas the control mice group received daily injections of 200 $\mu \mathrm{l}$ of sterile water for the same time period. $12 \mathrm{~h}$ after the last treatment blood samples were collected by cardiac puncture and analyzed as per the manufacturer's protocol in the assay kits procured from Span Diagnostics Ltd., Surat, India.

\subsection{Determination of in vivo antileishmanial activity}

To develop chronic experimental VL in a mice model, BALB/c mice (20-25 g) of either sex were injected with $200 \mu \mathrm{l}$ of sterile PBS containing $2 \times 10^{7} \mathrm{~L}$. donovani amastigotes of AG83 wildtype strains through the tail vein. The potency of the infection 30 days post infection was confirmed by spleen biopsies of randomly selected mice from each group. After that the treatment was initiated by intraperitoneal injections of $200 \mu \mathrm{l}$ of AmB and AmB-loaded nanoformulations at the dose regimen of $1 \mathrm{mg}$ per $\mathrm{kg}$ per day, for 5 consecutive days. One group of mice was kept as an infected control. On day 7 of the last dose, spleen impression smears were obtained and stained with Giemsa to count the number of amastigotes per 500 spleen cells. The parasite burden was determined by measuring the LDU (Leishman-Donovan Unit), which could be calculated by 
multiplying the number of counted amastigotes per 500 nuclei with the spleen weight in $\mathrm{mg} \cdot{ }^{32}$ The percentage of parasite suppression was calculated by the following formula:

$$
\% \text { parasite suppression }=(\mathrm{CC}-\mathrm{CT} / \mathrm{CC}) \times 100 \% \text {, }
$$

where CC is the count of amastigotes per 500 spleen cells of the infected controls and CT is the count of amastigotes per 500 spleen cells of the treated group.

\subsection{Statistical analysis}

Experimental data were expressed as mean \pm standard deviation. To determine the statistical significance, one way analysis of variance was used. When $P<0.01$ it was considered as significant whereas when $P>0.05$ then it was considered to be statistically insignificant

\section{Results}

\subsection{Spectral characterization of synthesized $O$-stearoyl mannose}

When the FTIR spectra of D-mannose and $O$-stearoyl mannose were compared (Fig. 2A) it was observed that in the case of Dmannose the intermolecular broad $\mathrm{O}-\mathrm{H}$ stretching was observed at $3200-3400 \mathrm{~cm}^{-1}$, but was almost absent in the case of OSM. The peaks in the region of $1260-1350 \mathrm{~cm}^{-1}$ denoted the

A

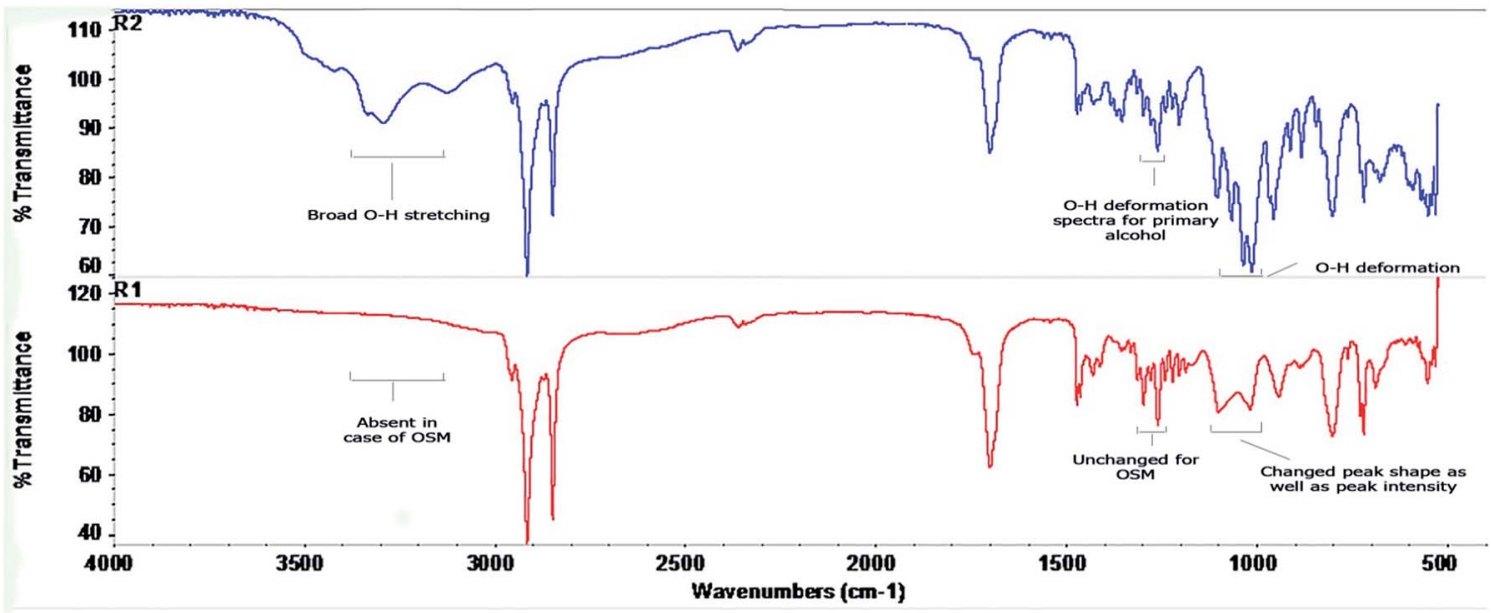

B
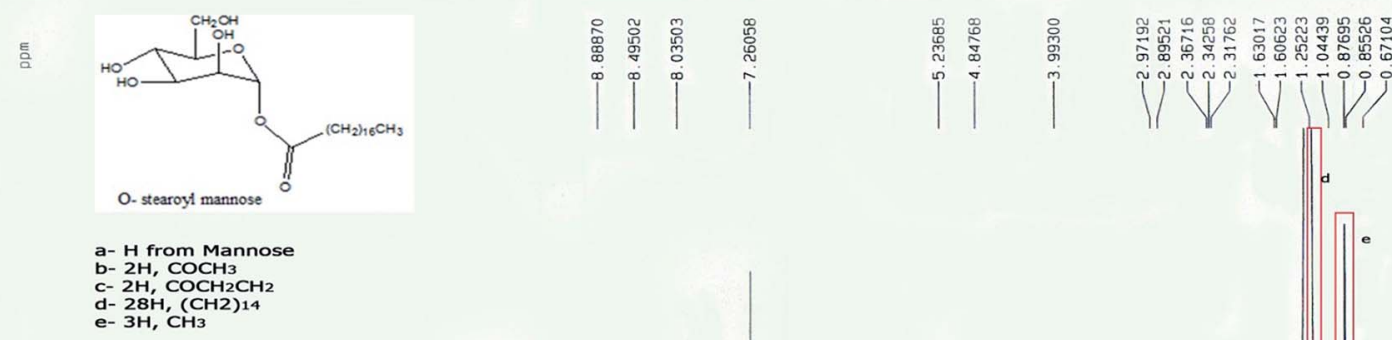

$2 \mathrm{H}_{1} \mathrm{COCH}_{2} \mathrm{CH}$

e- $3 \mathrm{H}, \mathrm{CH} 3$

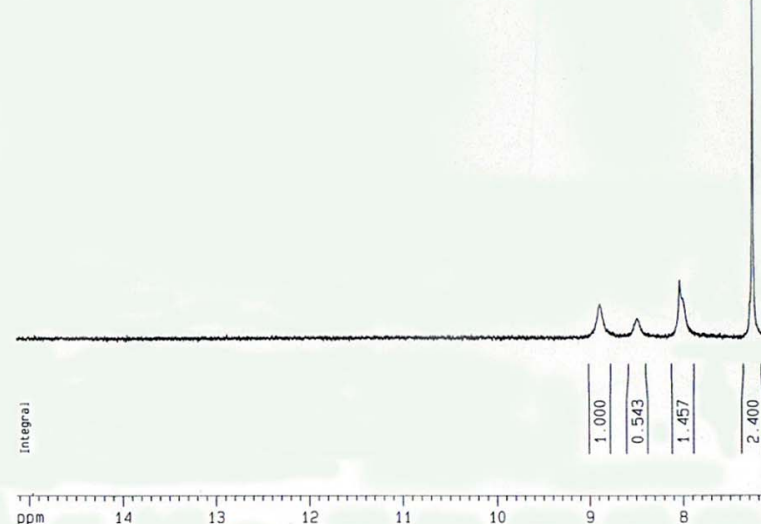

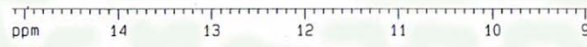

Fig. 2 (A) FTIR spectra of (R2) D-mannose and (R1) O-stearoyl mannose. (B) The ${ }^{1} \mathrm{H}$ NMR spectra of $O$-stearoyl mannose. 
O-H deformation spectra which were unchanged for both of them, whereas the shape of the $\mathrm{O}-\mathrm{H}$ deformation spectra at 1000-1100 $\mathrm{cm}^{-1}$ were completely changed for OSM. It was also observed that the ${ }^{-} \mathrm{CH}_{2}$ symmetric stretching at 2843-2863 $\mathrm{cm}^{-1}$ was almost the same for both but merely increased in intensity for OSM. For both of them sharp, asymmetric $>\mathrm{CH}_{2}$ stretching was found at $2916-2936 \mathrm{~cm}^{-1}$.

Fig. 2B depicted the following result:

${ }^{1} \mathrm{H}$ NMR (300 MHz, $\mathrm{CDCl}_{3}$ ): 5.23685-2.97192 ppm (H from mannose), $2.31762 \mathrm{ppm}\left(\mathrm{t}, 2 \mathrm{H}, \mathrm{COCH}_{3}\right), 1.60623 \mathrm{ppm}(\mathrm{m}, 2 \mathrm{H}$, $\left.\mathrm{COCH}_{2} \mathrm{CH}_{2}\right), 1.25223 \mathrm{ppm}\left(\mathrm{m}, 28 \mathrm{H},\left(\mathrm{CH}_{2}\right)_{14}\right), 0.87695 \mathrm{ppm}(\mathrm{t}$, $\left.3 \mathrm{H}, \mathrm{CH}_{3}\right)$.

\subsection{FTIR study}

The drug-excipient interactions, if any, could be efficiently determined by FTIR spectroscopic analysis. In Fig. 3C, the FTIR spectra for AmB showed asymmetric stretching at 2936-2916 $\mathrm{cm}^{-1}$ for long alkane chains. Non-conjugated $\mathrm{C}=\mathrm{C}$ stretching was observed at about $1680 \mathrm{~cm}^{-1}$. A peak near $1100 \mathrm{~cm}^{-1}$ was shown for $\mathrm{C}-\mathrm{O}$ stretching of the cyclic six-membered aromatic ring structure present in AmB. A broad peak appeared at 3416$3382 \mathrm{~cm}^{-1}$ for $\mathrm{N}-\mathrm{H}$ stretching of the primary aromatic amine. In the FTIR spectra of PVA (Fig. 3B), C-O stretching vibration and O-H deformation peaks were found at 1440-1395 $\mathrm{cm}^{-1}$ and 1320-1211 $\mathrm{cm}^{-1}$ respectively. C-H stretching vibrations for the $-\mathrm{CH}_{3}$ group were observed near $2950 \mathrm{~cm}^{-1}$ whereas a broad peak in between $3300 \mathrm{~cm}^{-1}$ to $3400 \mathrm{~cm}^{-1}$ was for $\mathrm{O}-\mathrm{H}$ stretching vibrations. All important peaks were present in the physical mixture of PLGA + PVA + AmB (Fig. 3D) with a few minor shifts. In the case of the blank nanoparticles no peaks for AmB were present. $-\mathrm{CH}_{2}$ symmetric stretching vibrations at $2850 \mathrm{~cm}^{-1}$ and sharp asymmetric $-\mathrm{CH}_{2}$ stretching at $2920 \mathrm{~cm}^{-1}$ were the major identifiable peaks of OSM which were present in both the spectra of the physical mixture of PLGA + PVA + AmB + OSM (Fig. 3G) and the AmB-loaded PLGA-OSM nanoparticles, with minor shifting due to the presence of van der Waal's attractions, formation of hydrogen bonding or any dipole-dipole interaction. $^{33}$
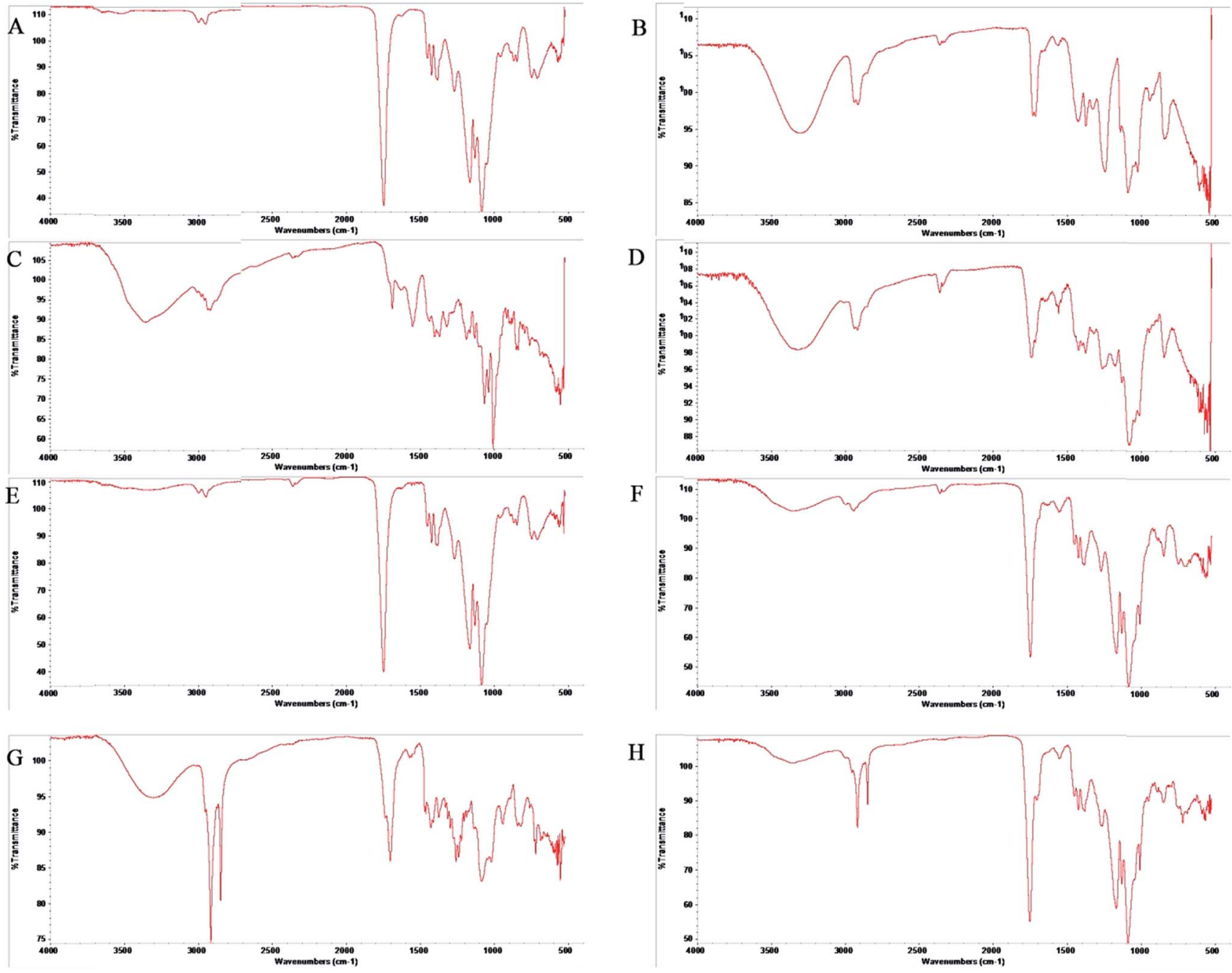

Fig. 3 A comparative FTIR analysis of (A) PLGA; (B) PVA; (C) AmB; (D) a physical mixture of PLGA + PVA + AmB; (E) blank nanoparticles; (F) AmBloaded PLGA nanoparticles; (G) a physical mixture of PLGA + PVA + AmB + OSM and (H) AmB-loaded PLGA-OSM nanoparticles. 

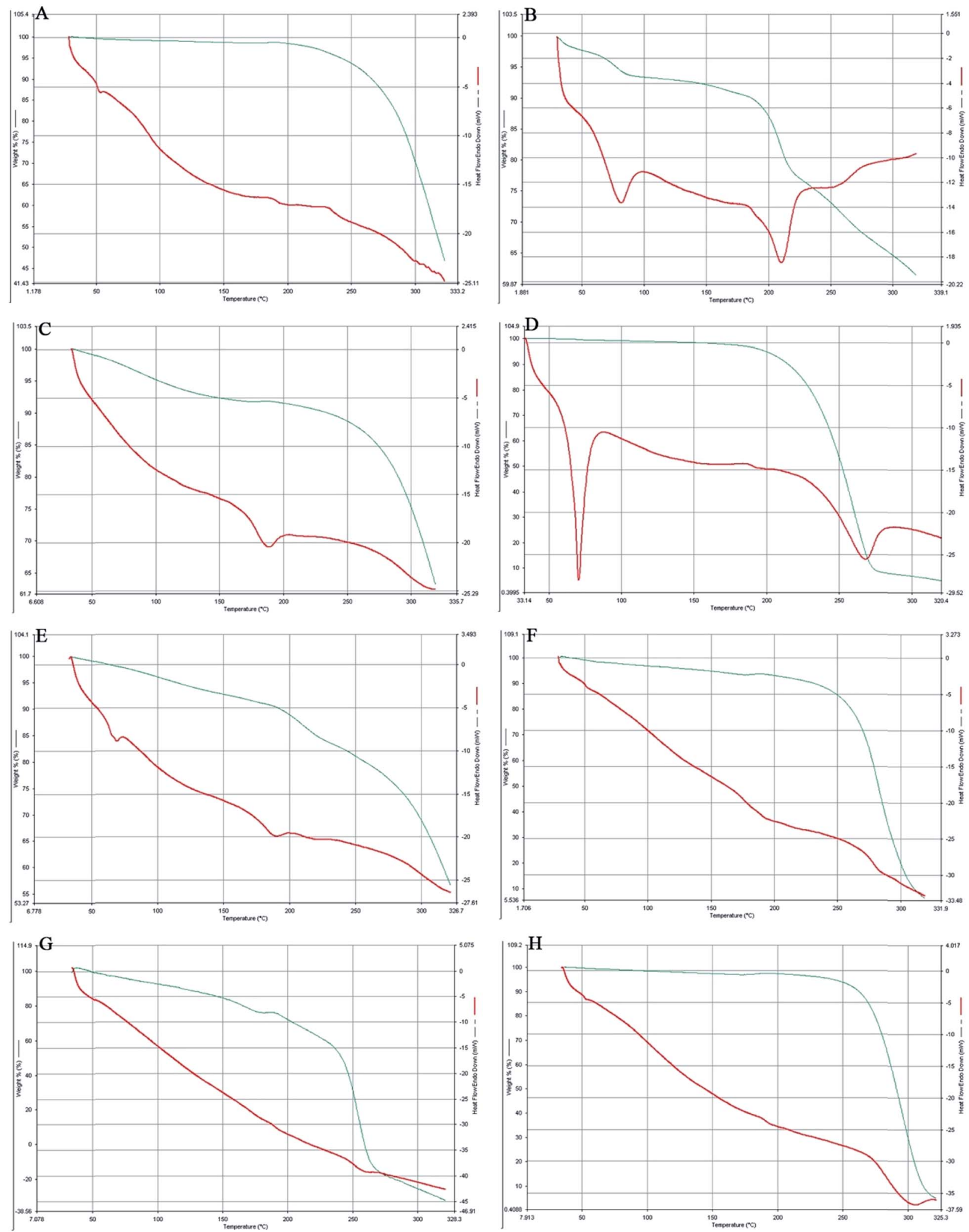

Fig. 4 A comparative study of differential scanning calorimetry and the thermogravimetric analysis of (A) PLGA; (B) AmB; (C) a physical mixture of PLGA + AmB + PVA; (D) OSM; (E) a physical mixture of PLGA + AmB + PVA + OSM; (F) AmB-loaded PLGA nanoparticles; (G) AmB-loaded PLGAOSM nanoparticles and $(\mathrm{H})$ blank nanoparticles. 


\section{3. $\quad$ DSC and TGA study}

The comparative study of the DSC thermograms of Fig. 4 consisted of PLGA, AmB, a physical mixture of PLGA + AmB + PVA,
OSM, a physical mixture of PLGA + AmB + PVA + OSM, AmBloaded PLGA nanoparticles, AmB-loaded PLGA-OSM nanoparticles and blank nanoparticles. It can be observed in Fig. 4B that $\mathrm{AmB}$ had two endothermic peaks at $80.08{ }^{\circ} \mathrm{C}$ and $210.14{ }^{\circ} \mathrm{C}$

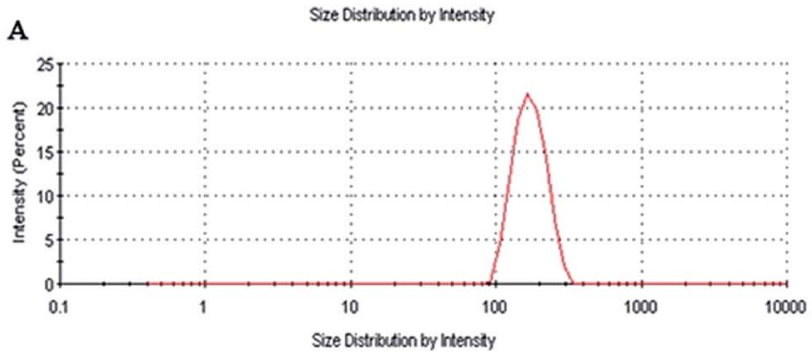

B

Size Distribution by intensity

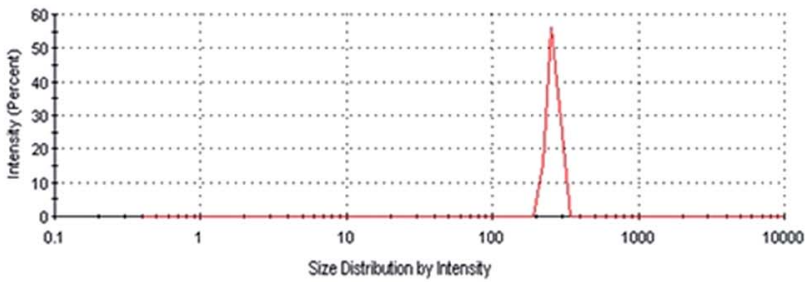

C
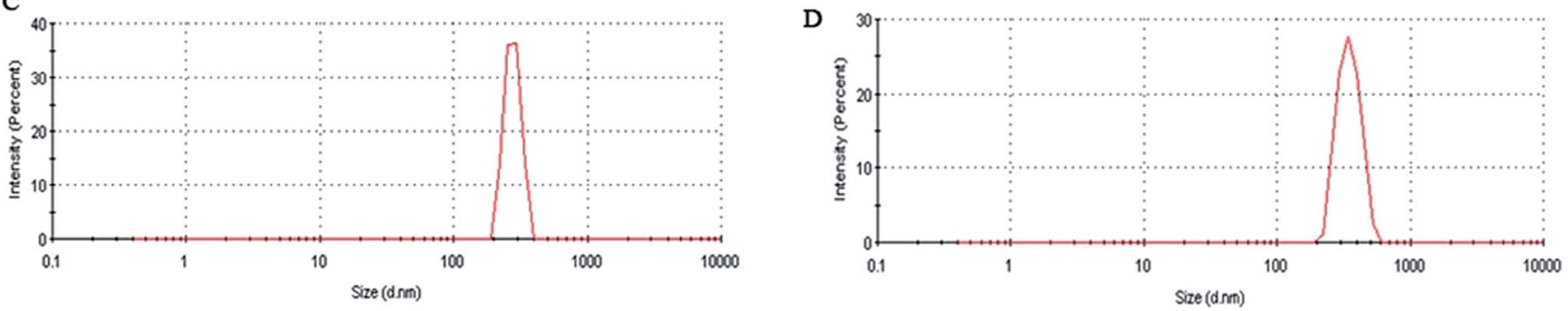

Fig. 5 A particle size distribution study of (A) AN1, (B) AN2, (C) AN3 and (D) ASMN.
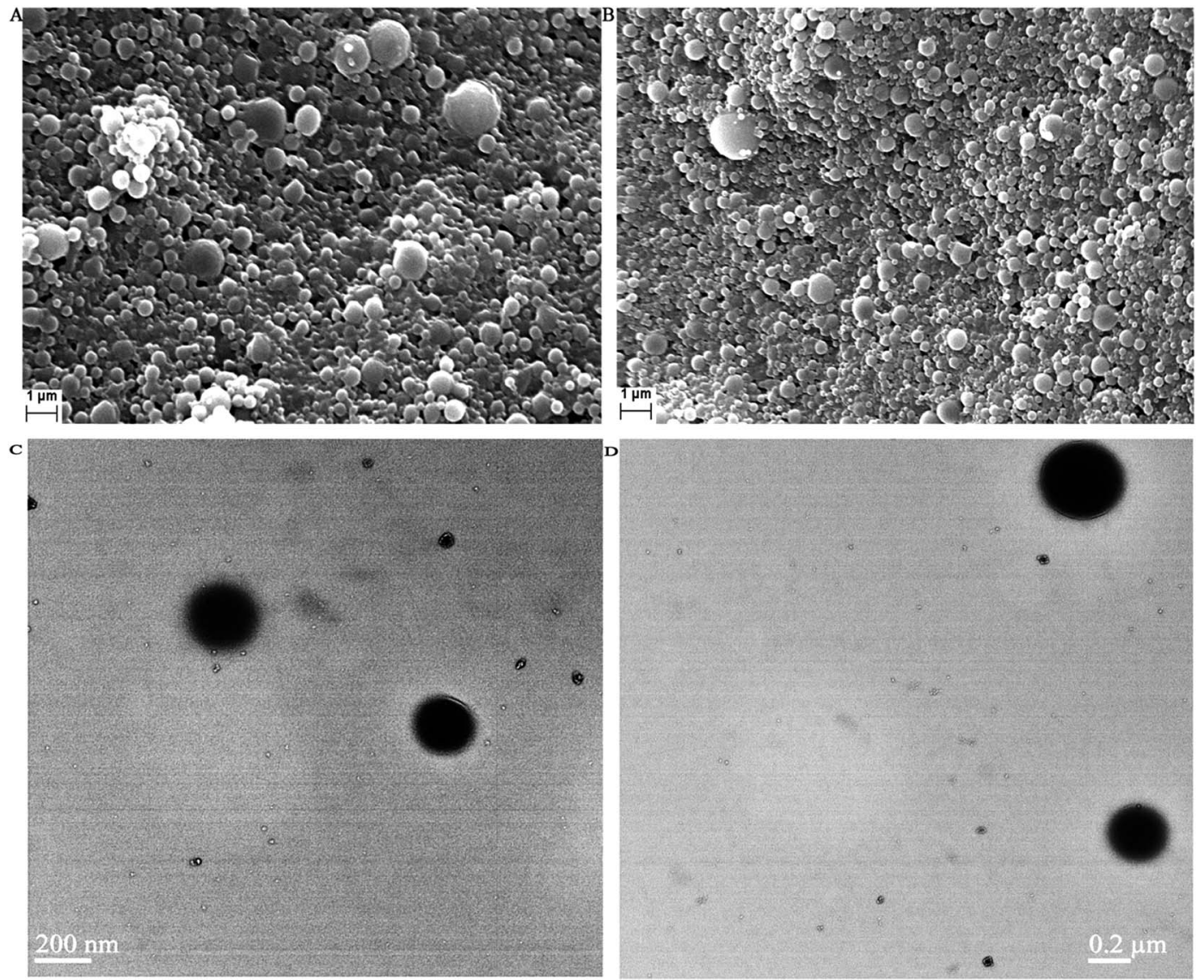

Fig. 6 Scanning electron microscopic images of (A) AmB-loaded PLGA nanoparticles and (B) AmB-loaded PLGA-OSM nanoparticles; and transmittance electron microscopic images of (C) AmB-loaded PLGA nanoparticles and (D) AmB-loaded PLGA-OSM nanoparticles. 


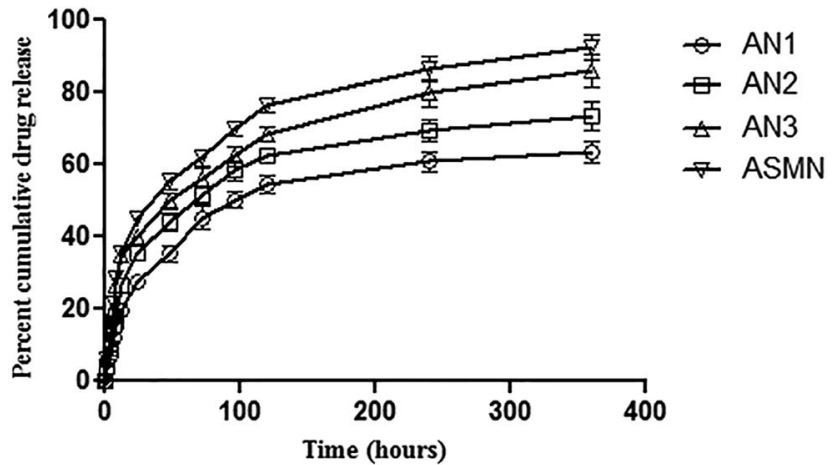

Fig. 7 An in vitro cumulative AmB release study of the formulations AN1, AN2, AN3 and ASMN.

and that the physical mixture of PLGA + AmB + PVA showed a single endothermic peak at $185.03^{\circ} \mathrm{C}$ (Fig. 4C). OSM (Fig. 4D) showed endothermic peaks at $70.59{ }^{\circ} \mathrm{C}$ and $267.13{ }^{\circ} \mathrm{C}$, whereas the physical mixture with drug and other excipients showed endothermic peaks at $66.08{ }^{\circ} \mathrm{C}$ and $187.83{ }^{\circ} \mathrm{C}$. Fig. $4 \mathrm{~F}$ and G represent the endothermic peak at $181.14{ }^{\circ} \mathrm{C}$ for AmB-loaded PLGA nanoparticles, and $177.25{ }^{\circ} \mathrm{C}$ and $257.64{ }^{\circ} \mathrm{C}$ for AmBloaded PLGA-OSM nanoparticles, whereas the blank nanoparticles showed an endothermic peak at about $300.09{ }^{\circ} \mathrm{C}$.

\subsection{Particle size, zeta potential and PDI}

From Table 1 and Fig. 5 it is observed that the average particle size of the formulated nanoparticles is $182.8 \pm 4.2 \mathrm{~nm}, 261.2 \pm$ $5.7 \mathrm{~nm}, 280.9 \pm 3.6 \mathrm{~nm}$ and $340.5 \pm 6.2 \mathrm{~nm}$, with PDI values of $0.355,0.510,0.686$ and 0.438 for AN1, AN2, AN3 and ASMN, respectively. It was also found from Table 1 that all of the nanoformulations consist of a negative zeta potential i.e. -11.8 $\pm 2.1 \mathrm{mV}$ for AN1, $-12.3 \pm 1.8 \mathrm{mV}$ for AN2, $-14.7 \pm 2.7 \mathrm{mV}$ for AN3 and $-24.9 \pm 1.6 \mathrm{mV}$ for ASMN nanoparticles.

\subsection{SEM and TEM studies}

Fig. 6 shows the SEM and TEM images of AmB-loaded PLGA nanoparticles and AmB-loaded PLGA-OSM nanoparticles. Those studies were done to evaluate the morphological characteristics.

\subsection{Drug loading study}

To determine the AmB loading in different types of formulations, firstly a standard curve of $\mathrm{AmB}$ was prepared by a liquid chromatographic technique. When the acquired areas were plotted against the respective concentrations a straight line was generated with an equation of $y=50.45 x-7.654\left(R^{2}=0.999\right)$. Using this equation the drug loading of different types of nanoformulations were calculated. From Table 1 it was found that for AN1, AN2 and AN3 the percentages of AmB loading were $6.7 \pm 0.5 \%, 9.0 \pm 0.5 \%$ and $11.5 \pm 0.7 \%$, respectively, and for ASMN it was found to be $10.2 \pm 0.8 \%$.

\subsection{AmB release study: in vitro}

An in vitro cumulative drug release study was carried out for $360 \mathrm{~h}$ and from Fig. 7 it was found that $63.48 \% \pm 1.08 \%$ of AmB was released from the formulation AN1, $73.45 \% \pm 1.54 \%$ from AN2, $86.19 \% \pm 1.86 \%$ from AN3 and from the formulation ASMN it was $92.56 \% \pm 2.05 \%$.

Table 2 displays the different release kinetic models to depict the drug release mechanism. It was observed that the all of the formulations (AN1, AN2, AN3 and ASMN) fit best in the Korsmeyer-Peppas model with the release exponent of 0.523, 0.491, 0.442 and 0.442 , respectively.

\subsection{Stability studies}

Storage stability studies of the experimental formulations were carried out at $4{ }^{\circ} \mathrm{C}$ and room temperature, for 6 months. At each predetermined time point $(1,7,15,30,60,90$ and 180 days) particle size and drug loading were evaluated. From Fig. 8 it was found that the increases in the average particle size of all four of the formulations were insignificant. Similarly the percentages of drug loading of the formulations were not decreased significantly within the stipulated period of time.

\subsection{Macrophage uptake study: ex vivo}

A macrophage uptake study was performed on isolated mice macrophages. Fig. 9 shows the comparative uptake study of two different types of FITC nanoparticles incubated for $10 \mathrm{~min}$ with macrophages. Fig. 9A-C show the bright, fluorescent and merged field images of the uptake of FITC-loaded PLGA nanoparticles whereas Fig. 9D-F show the bright, fluorescent and merged field images of the uptake of FITC-loaded PLGA-OSM nanoparticles.

\subsection{In vitro antileishmanial activity}

The drug sensitivities of AmB, the AmB-loaded PLGA nanoformulation (AN3) and the AmB-loaded PLGA-OSM

Table 2 Different models of release kinetics to evaluate the AmB release pattern

\begin{tabular}{lllllll}
\hline & \multicolumn{2}{l}{ Correlation coefficient $\left(R^{2}\right)$} & & & \\
\cline { 2 - 6 } Formulation & Zero order & First order & Higuchi & Hixon-Crowell & Korsmeyer-Peppas & $\begin{array}{l}\text { Release exponent } \\
(n)\end{array}$ \\
\hline AN1 & 0.683 & 0.781 & 0.912 & 0.749 & 0.744 & 0.523 \\
AN2 & 0.666 & 0.792 & 0.898 & 0.750 & 0.660 & 0.491 \\
AN3 & 0.664 & 0.855 & 0.889 & 0.791 & 0.629 & 0.442 \\
ASMN & 0.533 & 0.833 & 0.758 & 0.726 & 0.523 & 0.442
\end{tabular}


A

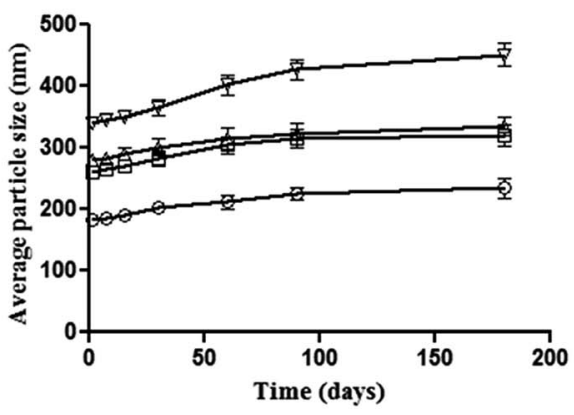

C

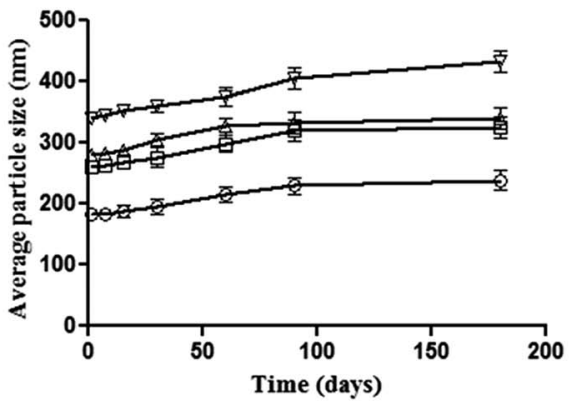

$\odot \mathrm{AN} 1$

— AN2

$\leftarrow \mathrm{AN} 3$

- ASMN

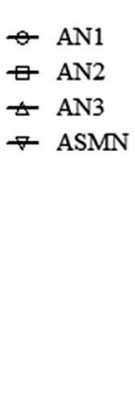

B

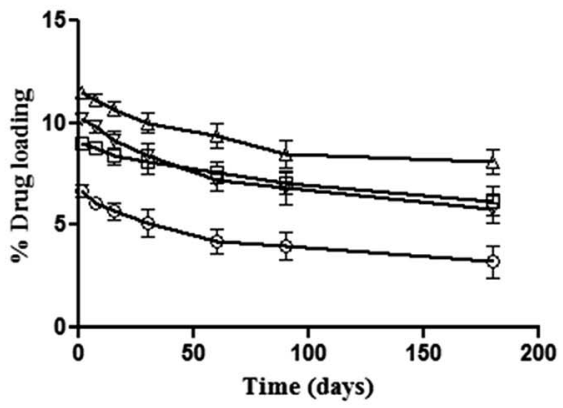

D

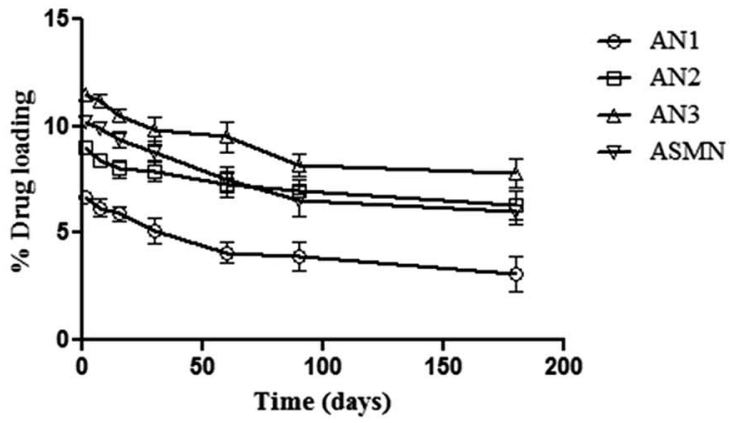

Fig. 8 A storage stability study of the formulated nanoparticles at $4{ }^{\circ} \mathrm{C}$ : (A) particle size analysis and (B) \% drug loading study, and at room temperature $\left(25^{\circ} \mathrm{C}\right)$ : (C) particle size analysis and (D) \% drug loading study.
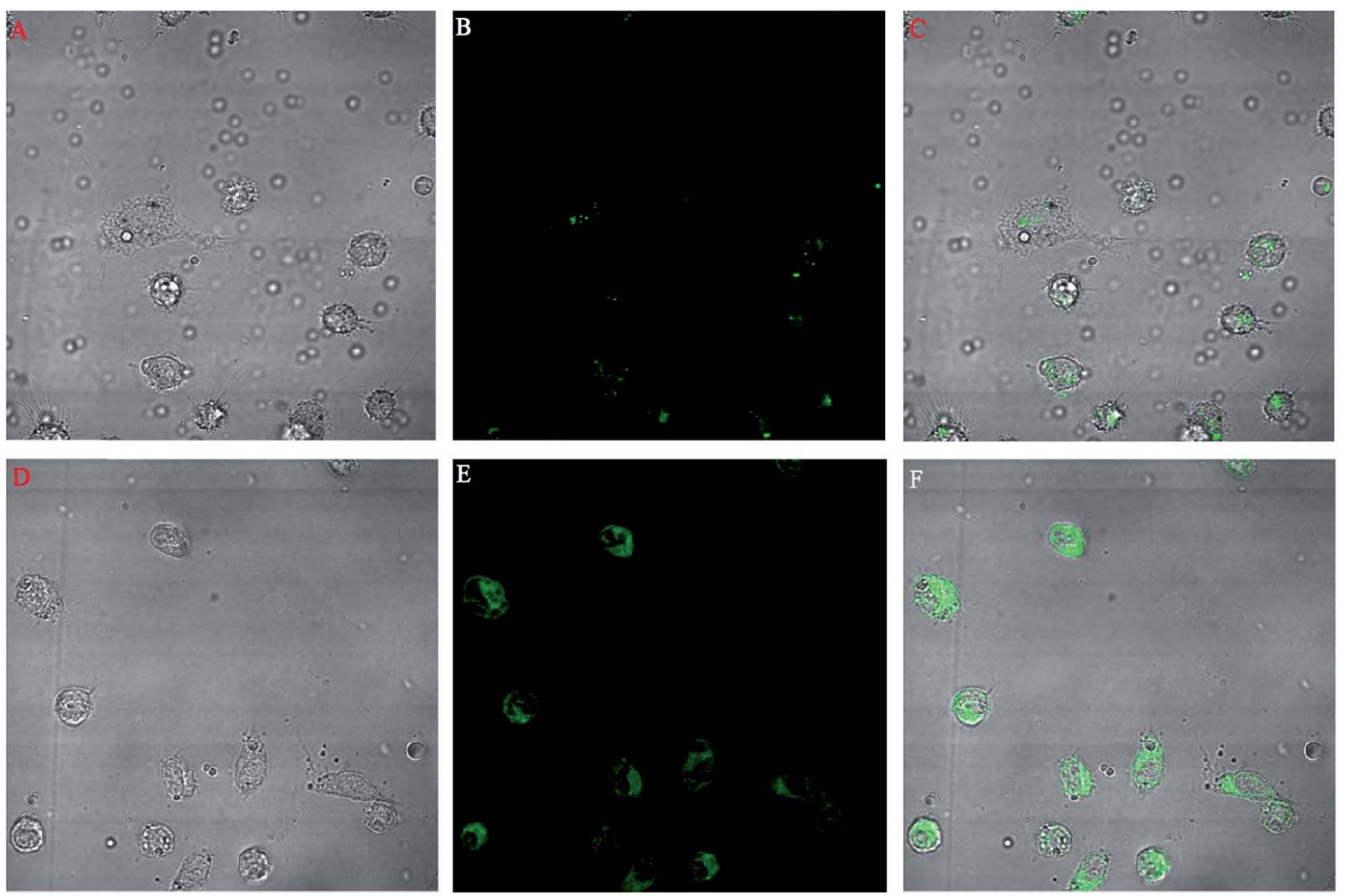

Fig. 9 The comparative macrophage uptake of FITC-loaded PLGA nanoparticles ((A) bright field, (B) fluorescent field and (C) merged field) and FITC-loaded PLGA-OSM nanoparticles ((D) bright field, (E) fluorescent field and (F) merged field) under a confocal microscope at 60× magnification.

nanoformulation (ASMN) were evaluated against Leishmania donovani AG83 wild type, drug resistant and GE1 field isolated axenic amastigotes, as well as cellular amastigotes. Table 3 shows the $\mathrm{IC}_{50}(50 \%$ inhibitory concentration) values against axenic amastigotes which reveal that the $\mathrm{IC}_{50}$ values were $0.21 \pm$ $0.08 \mu \mathrm{M}, 0.13 \pm 0.0 .9 \mu \mathrm{M}$ and $0.11 \pm 0.06 \mu \mathrm{M}$ for $\mathrm{AmB}, \mathrm{AN}$ and 
Table 3 The susceptibility of the drug or drug-loaded formulations against Leishmania donovani AG83 wild type, drug resistant and GE1 field isolated axenic amastigotes ${ }^{a}$

$\mathrm{IC}_{50}($ mean $\pm \mathrm{SD}, n=3) \mu \mathrm{M}$

Axenic amastigotes evaluation model

\begin{tabular}{|c|c|c|c|c|}
\hline Drug/formulation & Wild type & SSG-R & PMM-R & GE1 field isolate \\
\hline $\mathrm{AmB}$ & $0.21 \pm 0.08$ & $0.24 \pm 0.06$ & $0.27 \pm 0.0 .9$ & $0.22 \pm 0.07$ \\
\hline AmB-loaded PLGA nanoformulation (AN) & $0.13 \pm 0.09^{b}$ & $0.15 \pm 0.08^{b}$ & $0.17 \pm 0.07^{b}$ & $0.15 \pm 0.09^{b}$ \\
\hline AmB-loaded PLGA-OSM nanoformulation (ASMN) & $0.11 \pm 0.06^{b}$ & $0.13 \pm 0.09^{b}$ & $0.15 \pm 0.05^{b}$ & $0.14 \pm 0.06^{b}$ \\
\hline
\end{tabular}

${ }^{a}$ Assay methods are described in the Materials and methods section. ${ }^{b} P>0.05$, no significant difference compared with AmB in the case of all the strains.

Table 4 The susceptibility of the drug or drug-loaded formulations against Leishmania donovani AG83 wild type, drug resistant and GE1 field isolated cellular amastigotes ${ }^{a}$

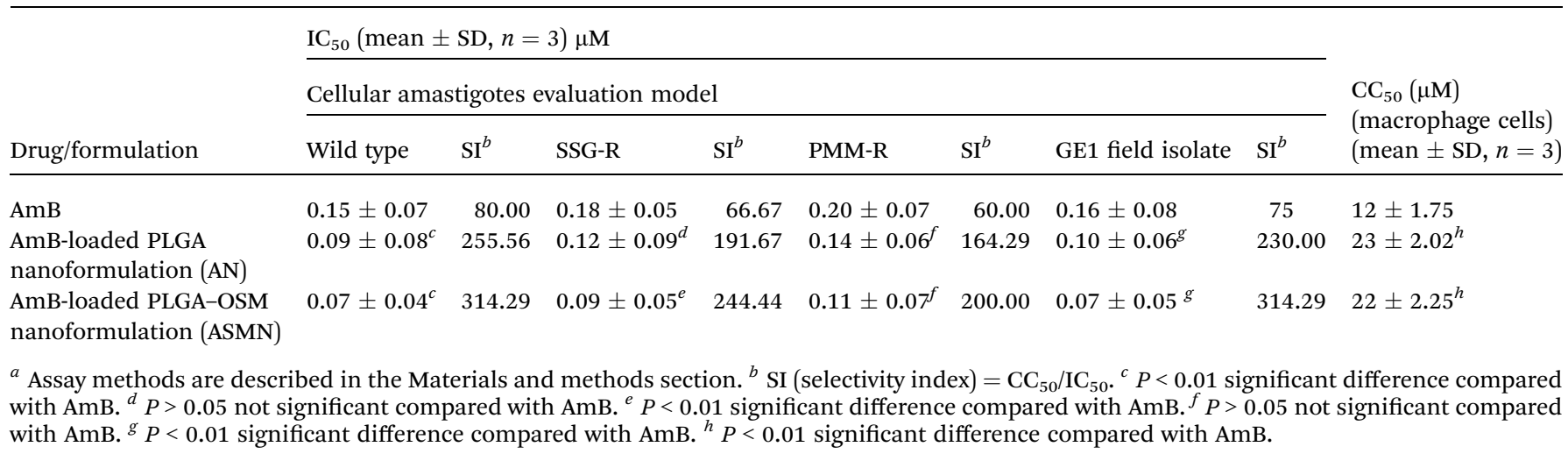

ASMN, respectively, against wild type AG83 strains; $0.24 \pm 0.06$ $\mu \mathrm{M}, 0.15 \pm 0.08 \mu \mathrm{M}$ and $0.13 \pm 0.09 \mu \mathrm{M}$ for AmB, AN and ASMN, respectively, against SSG-R strains; $0.27 \pm 0.0 .9 \mu \mathrm{M}, 0.17 \pm 0.07$ $\mu \mathrm{M}$ and $0.15 \pm 0.05 \mu \mathrm{M}$ for AmB, AN and ASMN, respectively, against PMM-R strains; and $0.22 \pm 0.07 \mu \mathrm{M}, 0.15 \pm 0.09 \mu \mathrm{M}$ and $0.14 \pm 0.06 \mu \mathrm{M}$ for AmB, AN and ASMN, respectively, against GE1 field isolated axenic amastigotes.

Table 4 displays the drug sensitivity against Leishmania donovani AG83 wild type, drug resistant and GE1 field isolated cellular amastigotes. It can be observed that against wild type cellular amastigotes the $\mathrm{IC}_{50}$ values were $0.15 \pm 0.07 \mu \mathrm{M}, 0.09 \pm$ $0.08 \mu \mathrm{M}$ and $0.07 \pm 0.04 \mu \mathrm{M}$ for AmB, AN and ASMN, respectively; for SSG-R these were $0.18 \pm 0.05 \mu \mathrm{M}, 0.12 \pm 0.09 \mu \mathrm{M}$ and $0.09 \pm 0.05 \mu \mathrm{M}$, for PMM-R $0.20 \pm 0.07 \mu \mathrm{M}, 0.14 \pm 0.06 \mu \mathrm{M}, 0.11$ $\pm 0.07 \mu \mathrm{M}$ and for GE1 field isolated cellular amastigotes these were $0.16 \pm 0.08 \mu \mathrm{M}, 0.10 \pm 0.06 \mu \mathrm{M}$ and $0.07 \pm 0.05 \mu \mathrm{M}$ for $\mathrm{AmB}, \mathrm{AN}$ and ASMN, respectively.

Table 4 also shows the calculated values of the selectivity index (SI) against different strains. These were 80.00, 255.56 and 314.29 for AmB, AN and ASMN, respectively, for wild type strains; 66.67, 191.67 and 244.44 for AmB, AN and ASMN, respectively, for SSG-R strains; 60.00, 164.29 and 200.00 for $\mathrm{AmB}, \mathrm{AN}$ and ASMN, respectively, for PMM-R strains and 75.00, 230.00 and 314.29 for GE1 field isolated strains for AmB, AN and ASMN, respectively.

\subsection{Biochemical markers associated toxicity assessment}

Fig. 11 shows the comparative toxicity study of the formulated nanoparticles i.e. AN and ASMN. It is observed from Fig. 11 that

Table 5 In vivo antileishmanial activity of amphotericin B and its formulations against $L$. donovani amastigote infected mice ${ }^{a}$

\begin{tabular}{|c|c|c|c|c|}
\hline & Control group & AmB treated group & AN treated group & ASMN treated group \\
\hline Spleen weight (mg) & $141.18 \pm 13.42$ & $119.56 \pm 11.27$ & $104.08 \pm 11.17$ & $98.04 \pm 7.83$ \\
\hline Amastigotes/500 nuclei $\pm \mathrm{SD}$ & $2498.40 \pm 451.54$ & $215.60 \pm 35.65$ & $75.80 \pm 8.61$ & $44.80 \pm 8.29$ \\
\hline $\mathrm{LDU}^{b} \times 10^{4} \pm \mathrm{SD}$ & $35.57 \pm 9.19$ & $2.60 \pm 0.62$ & $0.79 \pm 0.12^{c}$ & $0.44 \pm 0.11^{c}$ \\
\hline$\%$ of parasite suppression $\pm \mathrm{SD}$ & - & $91.31 \pm 1.10$ & $96.92 \pm 0.45^{d}$ & $98.19 \pm 0.33^{d}$ \\
\hline
\end{tabular}

${ }^{a}$ Assay methods are described in the Materials and methods section. ${ }^{b}$ LDU (Leishman-Donovan Unit) $=$ no. of amastigotes per 500 nuclei $\times$ tissue weight (mg). ${ }^{c} P<0.01$ significant compared to AmB. ${ }^{d} P<0.01$ significant compared to AmB. 
the intravenous administration of both of the formulations elevated the levels of hepatic enzymes, whereas a slight elevation in the levels of the blood urea nitrogen and creatinine is observed for both of the formulations.

\subsection{Antileishmanial study: in vivo}

Table 5 indicates the antileishmanial efficiency of the formulated AmB-loaded nanoparticles over AmB alone. The percentage of suppression of the parasite burden in the spleen of $\mathrm{BALB} / \mathrm{c}$ mice was used to evaluate the antileishmanial potential of the formulated nanoparticles and it was found to be $91.31 \pm 1.10 \%$ for the AmB-treated mice group, $96.92 \pm 0.45 \%$ for the AmB-loaded PLGA nanoparticles (AN) treated group of mice and $98.19 \pm 0.33 \%$ for AmB-loaded PLGA-OSM nanoparticles (ASMN) treated mice group.

\section{Discussion}

The successful synthesis of OSM from D-mannose was evident from the comparative analysis of the FTIR spectra of D-mannose and OSM (Fig. 2A); and ${ }^{1} \mathrm{H}$ nuclear magnetic resonance study of OSM (Fig. 2B). It was found from Fig. 2A that intermolecular O-H stretching at $3200-3400 \mathrm{~cm}^{-1}$ was absent in the OSM spectrum, which could indicate that the stearoyl moiety was attached to all of the $\mathrm{OH}$ groups present in the mannose. On the other hand, it was observed from the comparative spectral analysis that $\mathrm{O}-\mathrm{H}$ deformation peaks at $1260-1350 \mathrm{~cm}^{-1}$ were unchanged for mannose and OSM. The peaks' appearance in this range is an indication of the presence of primary alcohols i.e. $-\mathrm{CH}_{2} \mathrm{OH}$. As this was unchanged, it could suggest that the stearoyl moiety wasn't attached to this particular position. On the other hand a change in the shape and the intensity of the peaks at $1000-1100 \mathrm{~cm}^{-1}$ was observed. Peaks at that range indicated the presence of a secondary alcohol in mannose which was altered in the case of OSM. The alteration in the shape and intensity of the peaks at that particular position signifies the attachment of a stearoyl moiety at those $\mathrm{OH}$ groups. However, according to Plusquellec et al.,$^{34}$ the $\mathrm{OH}$ group attached to the $\alpha$-anomeric carbon was the most preferable position for the attachment of the stearoyl moiety. Along with the FTIR data, the ${ }^{1} \mathrm{H}$ NMR spectra further confirmed the synthesis of OSM.

In this study we have prepared different types of AmB-loaded nanoparticles, i.e. AN1, AN2, AN3 and ASMN. The major difference among the AN1, AN2 and AN3 formulations was the drug and polymer ratios for their preparations, whereas ASMN was an OSM surface decorated AmB-loaded PLGA nanoformulation. Before further physicochemical characterization of those formulated nanoparticles, a drug-excipient compatibility study was carried out to identify whether they were compatible with each other both before and after the formulations. According to the many formulation scientists throughout the world, FTIR is the best analytical approach to evaluate the drug-excipient interactions in the formulations and by performing FTIR it was observed that they were compatible with each other and that they didn't take part in any chemical interactions when physically mixed together or during the formulation. Due to the formation of hydrogen bonding, dipole-dipole interaction or van der Waals force there was some minor shifting of formulation which might be an indication of the physical interaction between them. ${ }^{35}$ As drug concentration was the only variable in the AN1, AN2 and AN3 formulations, we had chosen AN3 for the preformulation study (as well as the other studies) because it had the highest amount of $\mathrm{AmB}$ encapsulated in it. In the FTIR spectra of both of the formulations (Fig. $3 \mathrm{~F}$ and $\mathrm{H}$ ) the major peaks for $\mathrm{AmB}$ were absent, which indicated the proper encapsulation of it into the polymeric scaffold of PLGA and there was no free drug on the surface of the formulations. From the FTIR analysis it was also assumed that OSM was present on the surface of the ASMN formulation as the major identifiable peaks were present in both the spectra of the physical mixture of the drug and the excipients (Fig. 3F) and the formulation (Fig. 3H).

DSC-TGA analysis was further employed to confirm the drug-excipient compatibility. It was observed from the study that the drug AmB consisted of two endothermic peaks. The characteristic endothermic peak at $80.08^{\circ} \mathrm{C}$ was for dehydration or loss of water which was supported by the TGA analysis, and decomposition was started at $195.05{ }^{\circ} \mathrm{C}$ followed by melting at $210.14{ }^{\circ} \mathrm{C}$. The synthesized OSM shows a sharp endothermic peak at $70.59{ }^{\circ} \mathrm{C}$, which could be the result of initial melting of the stearic acid part in OSM, followed by decomposition starting at $230.28{ }^{\circ} \mathrm{C}$ and melting at $267.13{ }^{\circ} \mathrm{C}$. The presence of endothermic peaks at $181.14{ }^{\circ} \mathrm{C}$ and $177.25{ }^{\circ} \mathrm{C}$, respectively, in the case of AmB-loaded PLGA nanoparticles and AmB-loaded PLGA-OSM nanoparticles depicted the presence of AmB in the polymeric core of the formulation, whereas in the case of AmB-loaded PLGA-OSM nanoparticles, the presence of an endothermic peak at $257.64{ }^{\circ} \mathrm{C}$ indicates the presence of OSM in the formulation.

Particle size had an enormous role in the biodistribution of the pharmaceuticals which in turn influenced the biological activity of the same. ${ }^{36}$ From Table 1 and Fig. 5 it could be found that all of the formulated nanoparticles were in the nanosize range. The formulations of AN1, AN2 and AN3 possessed an almost similar average particle size, whereas the mannosemodified AmB-loaded PLGA nanoparticles (ASMN) had a slightly higher average particle diameter, which might be due to the $O$-stearoyl mannose associated modification. PDI values indicated the heterogeneity of the particles in a dispersion. From the PDI values of the formulations it could be signified that a narrow nanosize distribution of particles was present in all of the formulations. The zeta potential indicated the load of the surface charge of the particles. The negative values of the zeta potential within around $25 \mathrm{mV}$ ensured the physical stability of all of the nanoformulation systems. The balance between the repulsive and attractive force determined the stability of the system. Whenever the force of attraction was getting high, the particles became aggregated which could be prevent by high repulsive force. It was noticed that nanoparticles with a zeta potential of approximately $\pm 30 \mathrm{mV}$ gained stability in suspension. ${ }^{37,38}$ 
To evaluate the morphological pattern of the formulated nanoparticles of both types, SEM and TEM studies were carried out and these showed that the particles of the formulation of both types i.e. AmB-loaded PLGA nanoparticles and AmBloaded PLGA-OSM nanoparticles were spherical in shape with smooth surfaces. From the SEM study it was also observed that the nanoparticles were slightly polydispersed as also depicted by their PDI values.

When the AmB loadings onto the formulated AN1, AN2, AN3 and ASMN nanoparticles were evaluated it was found that among AN1, AN2 and AN3, AN3 possessed the highest AmB loading of $11.5 \pm 0.7 \%$. It was also observed that the drug loading was increased with the increasing amount of AmB during formulation. In the case of ASMN it was $10.2 \pm 0.8 \%$ which was almost similar to the loading of AN3.

The evaluation of the in vitro release study of a formulation is important as the biological fate of the formulation depends on it. When the AmB in vitro cumulative release study was evaluated, it was observed that the formulation ASMN released the highest amount of $\mathrm{AmB}(92.56 \% \pm 2.05 \%)$ during the prolonged time period. Among the formulations AN1, AN2 and AN3; AN3 released highest amount of encapsulated AMB $(86.19 \% \pm$ $1.86 \%$ ). In Fig. 7 the drug release patterns of AmB from different formulations are presented. From there it could be assumed that the initial release of $\mathrm{AmB}$ happened due to the presence of the drug nearer to the polymeric surface, whereas the slower release of AmB was due to the diffusion of the drug from the polymeric core of the formulations. From Table 2, the drug release kinetics of the four different experimental formulations on different release kinetics models could be observed and it was found that all of the formulations of AmB-loaded nanoparticles, AN1, AN2, AN3 and ASMN, followed the KorsmeyerPeppas release kinetics model as evident from their values of correlation coefficient $\left(R^{2}\right)$ and it was also found that the values of release exponent $(n)$ of all the formulations were between 0.43 and 0.85 , indicating the anomalous diffusion or non-Fickian diffusion of the AmB from the hydrophobic polymeric core of the formulated nanoparticles. ${ }^{39}$

Stability on storage is a very important parameter for pharmaceuticals. To evaluate the storage stability, experimental formulations of four different types were kept at $4{ }^{\circ} \mathrm{C}$ and room temperature for 6 months. At each predetermined time point sample aliquots were collected to evaluate the particle size and drug loading. Insignificant changes were observed in both cases for all of the types of formulations, which indicated that the formulated nanoparticles were stable in these particular storage conditions.

The promastigote stage of leishmania parasites are phagocytosed by macrophages, and within the phagolysosomal vacuoles they convert to the amastigote stage and multiply to show pathogenicity in the host. Thus it is important to target the macrophages to increase the antileishmanial efficacy of the antileishmanial drugs. We prepared PLGA-OSM nanoparticles which were mannose-modified PLGA nanoparticles to target the macrophages. In this regard, ex vivo macrophage uptake studies of FITC-loaded PLGA nanoparticles and FITC-loaded PLGAOSM nanoparticles were carried out. From Fig. 9D-F it could be observed that FITC-loaded PLGA-OSM nanoparticles were better taken up by the macrophages than the surface unmodified FITC-loaded PLGA nanoparticles, which indicated that the surface modification by mannose in formulated nanoparticles influenced the increase in the uptake by the macrophages.

In the present scenario of antileishmanial chemotherapy, AmB is a gold standard and used to treat leishmaniasis (especially the visceral one) throughout the world. The liposomal formulation of AmB is currently being used, but in the search for a more specific AmB delivery system with minimal toxicity or adverse effects, we have developed a mannose modified PLGA encapsulated AmB nanoformulation. To evaluate the antileishmanial potency of the formulated nanoparticles, an in vitro drug sensitivity assay was carried out against L. donovani AG83 wild type, SSG resistant, PMM resistant and GE1 field isolated strains. $50 \%$ inhibitory concentrations $\left(\mathrm{IC}_{50}\right)$ were determined against both axenic and cellular amastigotes and tabulated in Tables 3 and 4, respectively. The $\mathrm{CC}_{50}$ values were determined for free $\mathrm{AmB}, \mathrm{AN}$ and $\mathrm{ASMN}$ against isolated mice peritoneal macrophages and also tabulated in Table 4. A drug substance with intended biological activity should possess enough balance between efficacy for its intended purpose and its safety, which could be expressed as its selectivity index (SI). The AmB had an adequate safety and efficacy ratio which could be found from its SI values tabulated in Table 4. Evaluating the SI values of both types of formulations against different amastigote strains (Fig. 10), it was noticed that the mannose-modified formulation of AmB (ASMN) having a greater safety and efficacy balance means that it has higher SI values against wild type, drug resistant and GE1 field isolated amastigotes than only AmB-loaded PLGA nanoparticles.

The results of the in vivo toxicity assessment (Fig. 11) showed that the level of the hepatic enzymes, i.e. AST and ALT, were increased for both types of formulations but not so that they resulted in hepatotoxicity. It was also observed that the BUN and creatinine levels were not significantly elevated, which in turn reflected that the formulations of both types were not nephrotoxic at that particular dose regimen.

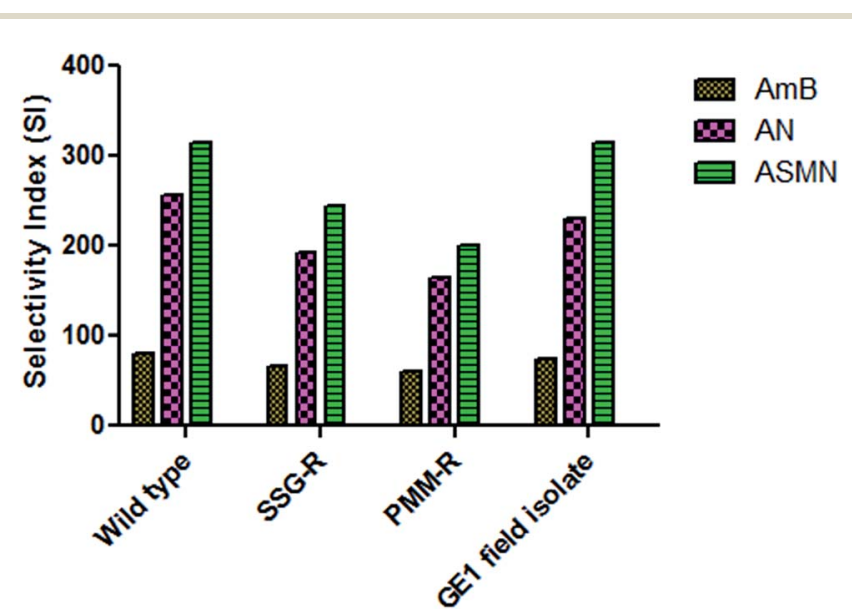

Fig. 10 A comparative study of the $\mathrm{SI}$ of $A m B, A N$ and $A S M N$ against AG83 wild type, SSG-R, PMM-R and GE1 strains. 


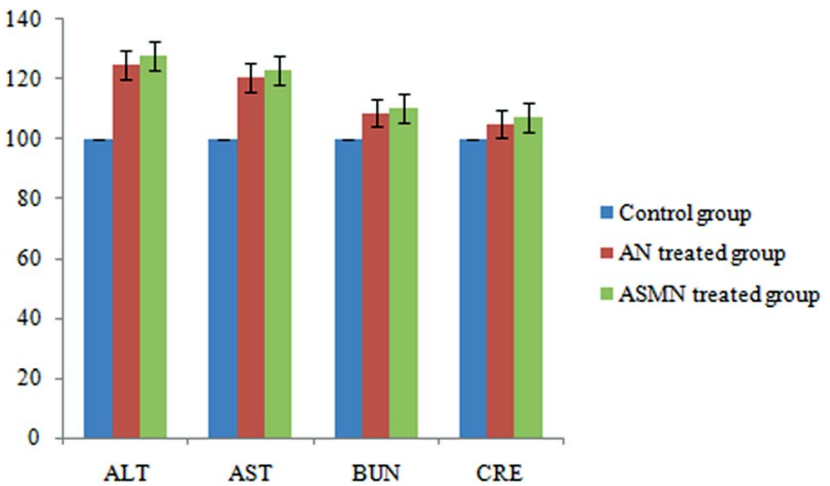

Fig. 11 An in vivo toxicity assessment of formulated nanoparticles by biochemical markers.

The in vivo antileishmanial potency of the free AmB and both types of nanoformulations were evaluated on a chronic VL infected mice model according to Mullen et al. ${ }^{40}$ It was observed from Table 5 that the mannose modified AmB-loaded PLGAOSM nanoparticles exerted superior activity over free AmB as well as over AmB-loaded nanoparticles. This was might be due to the mannose modification of the nanoformulation, which could easily be taken up by the macrophages, as is already evident from the ex vivo macrophage uptake study.

\section{Conclusion}

This experimental work was a fruitful approach to fabricate an amphotericin B-loaded mannose decorated PLGA nanoparticulate associated drug delivery system for the treatment of the most endemic form of leishmaniasis, VL, also known as kala-azar in India. From this work it could be concluded that the mannose decorated formulation of PLGA is more efficacious than the unmodified drug-loaded PLGA nanoparticles against wild type, field isolated, as well as drug resistant amastigotes of L. donovani in vitro and in the case of the in vivo antileishmanial evaluation, the effectiveness of the mannose modified PLGA nanoparticles exerted superior efficacy. This delivery system reflects its ability for suitable homing of the drugs used in the treatment of macrophage associated diseases like visceral leishmaniasis.

\section{Conflict of interest}

The authors have no conflict of interest.

\section{Acknowledgements}

This experimental work was supported by the Indian Council of Medical Research (ICMR), India. The first author, Santanu Ghosh, is a senior research fellow of ICMR (BMS/FW/Nano/ 2014-26340/Feb.15/11/Govt.). We also acknowledge Dr Shyamol Roy, Scientist, Indian Institute of Chemical Biology, Kolkata, India for his valuable and generous support to obtain the Leishmania donovani AG83 (wild) strain.

\section{References}

1 World Health Organization (2014), Global Health Observatory Data - Leishmaniasis, available at: http:// www.who.int/gho/neglected_diseases/leishmaniasis/en/ GlobalHealthObservatory, accessed on April 21, 2015.

2 V. Field, P. Gautret, P. Schlagenhauf, G. D. Burchard, E. Caumes, M. Jensenius, F. Castelli, E. Gkrania-Klotsas, L. Weld, R. Lopez-Velez and P. de Vries, BMC Infect. Dis., 2010, 10, 330.

3 I. Okwor and J. E. Uzonna, Immunol. Res., 2013, 56, 163-171. 4 J. Alvar, I. D. Vélez, C. Bern, M. Herrero, P. Desjeux, J. Cano, J. Jannin and M. den Boer, PLoS One, 2012, 7, e35671.

5 N. C. Peters, J. G. Egen, N. Secundino, A. Debrabant, N. Kimblin, S. Kamhawi, P. Lawyer, M. P. Fay, R. N. Germain and D. Sacks, Science, 2008, 321, 970-974.

6 N. C. Peters and D. L. Sacks, Cell. Microbiol., 2009, 11, 12901296.

7 H. Laufs, K. Müller, J. Fleischer, N. Reiling, N. Jahnke, J. C. Jensenius, W. Solbach and T. Laskay, Infect. Immun., 2002, 70, 826-835.

8 G. van Zandbergen, M. Klinger, A. Mueller, S. Dannenberg, A. Gebert, W. Solbach and T. Laskay, J. Immunol., 2004, 173, 6521-6525.

9 M. M. Kane and D. M. Mosser, Curr. Opin. Hematol., 2000, 7, 26-31.

10 M. K. Basu and S. Lala, Curr. Mol. Med., 2004, 4, 681-689.

11 F. Ahsan, I. P. Rivas, M. A. Khan and A. I. Suárez, J. Controlled Release, 2002, 79, 29-40.

12 R. Sett, K. Sarkar and P. K. Das, J. Infect. Dis., 1993, 168, 994999.

13 N. P. Truong, M. R. Whittaker, C. W. Mak and T. P. Davis, Expert Opin. Drug Delivery, 2015, 12, 129-142.

14 N. P. Truong, J. F. Quinn, M. R. Whittaker and T. P. Davis, Poly. Chem., 2016, 7, 4295-4312.

15 S. Mondal, P. Roy, S. Das, A. Halder, A. Mukherjee and T. Bera, PLoS One, 2013, 8, e81492.

16 S. Asthana, A. K. Jaiswal, P. K. Gupta, A. Dube and M. K. Chourasia, Eur. J. Pharm. Biopharm., 2015, 89, 62-73.

17 P. H. Stahl and S. Gordon, J. Cell Biol., 1982, 93, 49-56.

18 S. Asthana, P. K. Gupta, A. K. Jaiswal, A. Dube and M. K. Chourasia, Pharm. Res., 2015, 32, 2663-2677.

19 M. E. Taylor and K. Drickamer, J. Biol. Chem., 1993, 268, 399404.

20 A. Mukhopadhyay and S. K. Basu. Biotechnology in India I, 2003, vol. 84, pp. 183-209.

21 S. Zhu, M. Niu, H. O'Mary and Z. Cui, Mol. Pharm., 2013, 10, 3525-3530.

22 L. H. Freitas-Junior, E. Chatelain, H. A. Kim and J. L. Siqueira-Neto, Int. J. Parasitol.: Drugs Drug Resist., 2012, 2, 11-19.

23 B. Mukherjee, K. Santra, G. Pattnaik and S. Ghosh, Int. J. Nanomed., 2008, 3, 487-496.

24 M. Niu, Y. W. Naguib, A. M. Aldayel, Y. C. Shi, S. D. Hursting, M. A. Hersh and Z. Cui, Mol. Pharm., 2014, 11, 4425-4436. 
25 J. Lamarra, S. Rivero and A. Pinotti, Design of chitosan-based nanoparticles functionalized with gallic acid, Mater. Sci. Eng., C, 2016, 67, 717-726.

26 A. W. Stadnyk, A. D. Befus and J. Gauldie, Characterization of nonspecific esterase activity in macrophages and intestinal epithelium of the rat, J. Histochem. Cytochem., 1990, 38, 1-6.

27 P. Roy, S. Das, T. Bera, S. Mondal and A. Mukherjee, Andrographolide nanoparticles in leishmaniasis: characterization and in vitro evaluations, Int. J. Nanomed., 2010, 5, 1113-1121.

28 K. Kar, K. Mukerji, K. Naskar, A. Bhattacharya and D. K. Ghosh, J. Protozool., 1990, 37, 277-279.

29 D. M. Iovannisci and B. Ullman, J. Parasitol., 1983, 69, 633636.

30 M. Nahar and N. K. Jain, Pharm. Res., 2009, 26, 2588.

31 A. A. Seymour, J. H. Sheldon, P. L. Smith, M. Asaad and W. L. Rogers, J. Pharmacol. Exp. Ther., 1994, 269, 263-270.

32 K. D. Manandhar, T. P. Yadav, V. K. Prajapati, S. Kumar, M. Rai, A. Dube, O. N. Srivastava and S. Sundar, J. Antimicrob. Chemother., 2008, 62(2), 376-380.
33 S. Basu, B. Mukherjee, S. Roy Chowdhury, P. Paul, R. Choudhury, A. Kumar, L. Mondal, M. H. Chowdhury and R. Maji, Int. J. Nanomed., 2012, 7, 6049-6061.

34 D. Plusquellec, F. Roulleau, F. Bertho, M. Lefeuvre and E. Brown, Tetrahedron, 1986, 42, 2457-2467.

35 P. J. Das, P. Paul, B. Mukherjee, B. Mazumder, L. Mondal, R. Baishya, M. C. Debnath and K. S. Dey, Mol. Pharm., 2015, 12, 2651-2664.

36 C. He, Y. Hu, L. Yin, C. Tang and C. Yin, Biomaterials, 2010, 31, 3657-3666.

37 V. J. Mohanraj and Y. Chen, Trop. J. Pharm. Res., 2006, 5, 561-573.

38 R. Fernandez-Urrusuno, P. Calvo, C. Remuñán-López, J. L. Vila-Jato and M. J. Alonso, Pharm. Res., 1999, 16, 1576-1581.

39 R. Maji, N. S. Dey, B. S. Satapathy, B. Mukherjee and S. Mondal, Int. J. Nanomed., 2014, 9, 3107.

40 A. B. Mullen, A. J. Baillie and K. C. Carter, Antimicrob. Agents Chemother., 1998, 42, 2722-2725. 